\title{
Aharonov-Bohm oscillations in the vortex dynamics in superconducting hollow cylinders
}

\author{
V. N. Gladilin \\ TQC, Universiteit Antwerpen, Universiteitsplein 1, B-2610 Antwerpen, Belgium, and \\ INPAC, Katholieke Universiteit Leuven, Celestijnenlaan 200D, B-3001 Leuven, Belgium \\ J. Tempere and J. T. Devreese \\ TQC, Universiteit Antwerpen, Universiteitsplein 1, B-2610 Antwerpen, Belgium, \\ V. V. Moshchalkov \\ INPAC, Katholieke Universiteit Leuven, Celestijnenlaan 200D, B-3001 Leuven, Belgium \\ (Received 19 March 2012; revised manuscript received 23 August 2012; published 13 September 2012)
}

\begin{abstract}
Using time-dependent Ginzburg-Landau theory we demonstrate that the Aharonov-Bohm (AB) effect, resulting from a Berry phase shift of the (macroscopic) wave function, is revealed through the dynamics of topological phase defects present in that same wave function. We study vortices and antivortices on the surface of a hollow superconducting cylinder, moving on circular orbits as they are subjected to the force from the current flowing parallel to the cylinder axis. Due to the AB effect the orbit deflections, caused by a magnetic field component along the cylinder axis, become periodic as a function of field, leading to strong and robust resistance oscillations.
\end{abstract}

DOI: 10.1103/PhysRevB.86.104508

PACS number(s): 74.25.Ha, 74.25.Uv, 74.25.Wx, 74.78.-w

\section{INTRODUCTION}

Recent advances in nanotechnology have triggered interest in experimental and theoretical studies of mesoscopic and nanoscale superconductivity on curvilinear surfaces. ${ }^{1-9}$ In superconducting spherical nanoshells the surface curvature leads to a Magnus-Lorentz force, which pushes the vortices and antivortices towards the opposite poles of the shell. This can be considered as an effective pinning of vortices and antivortices at the poles, which strongly affects both the equilibrium distributions of vortices and their dynamics. ${ }^{3,5,6}$ The effects of surface curvature on vortex dynamics have been recently analyzed also for curved stripes and hollow cylinders. ${ }^{89}$ An important aspect of hollow cylinders, which have made them a popular subject of study, is their doubly connected topology. The Aharonov-Bohm (AB) oscillations, ${ }^{10}$ originating from a shift of the geometric (Berry) phase ${ }^{11}$ of the charge-carrier wave function by an enclosed magnetic flux, were first experimentally observed in superconducting hollow cylinders ${ }^{12}$ and rings. ${ }^{13}$ In the present paper, we analyze the vortex dynamics in superconducting hollow cylinders, subjected to a magnetic field tilted with respect to the cylinder axis. In particular, we investigate the effect of the oscillating persistent current, related to the $\mathrm{AB}$ effect, on the vortex motion and the corresponding resistive state. The obtained results suggest that, in parallel to the critical temperature oscillations, ${ }^{13,14}$ also oscillations of the resistance, caused by vortex motion, can be used as a tool to probe the $A B$ persistent currents in superconducting hollow cylinders.

\section{MODEL}

The sketch of the structure under consideration is shown in Fig. 1(a). A thin superconducting hollow cylinder with thickness $d$, length $L$, and radius $R$ is subjected to an external homogeneous magnetic field $\mathbf{B}_{0}$, which in general has nonzero components both along the cylinder axis $\left(B_{0 \|}\right)$ and in the perpendicular direction $\left(B_{0 \perp}\right)$. In the chosen cylindrical coordinate frame, the $z$ axis coincides with the cylinder axis, while the direction of $B_{0 \perp}$ corresponds to the angular coordinate $\phi=\pi / 2$. At $z=0$ and $z=L$, where normalmetal/superconductor boundary conditions are applied, an external current with density $j_{e}$ is along the $z$ axis.

The vortex dynamics is described within the timedependent Ginzburg-Landau (TDGL) approach. For numerical simulations we use the implementation of this approach described in detail in Refs. 5,15 and applicable when the thickness of the superconductor is smaller than the GinzburgLandau (GL) coherence length $\xi$, with obvious adaptations to the case of a cylindrical layer. Like in Ref. 15, the relevant quantities are made dimensionless by expressing lengths in units of $\sqrt{2} \xi$, time in units of $\pi \hbar /\left[4 k_{B}\left(T_{c}-T\right)\right] \approx 11.6 \tau_{\mathrm{GL}}$, magnetic field in units of $\Phi_{0} /\left(4 \pi \xi^{2}\right)=H_{c 2} / 2$, current density in units of $\Phi_{0} /\left(2 \sqrt{2} \pi \mu_{0} \lambda^{2} \xi\right)=3 \sqrt{3} /(2 \sqrt{2}) j_{c}$, and scalar potential in units of $2 k_{B}\left(T_{c}-T\right) /(\pi e)$. Here, $\Phi_{0}=\pi \hbar / e$ is the magnetic flux quantum, $\mu_{0}$ is the vacuum permeability, $\lambda$ is the penetration depth, $\tau_{\mathrm{GL}}$ is the GL time, $H_{c 2}$ is the second critical field, and $j_{c}$ is the critical (depairing) current density of a thin wire or film.${ }^{16}$ The results, described below, are obtained for a fixed value of the GL parameter $(\kappa=0.77)$. Note that this particular choice of $\kappa$ is not really restrictive: with the used units, the parameter $\kappa$ explicitly enters the equations only through the expression $\mathbf{A}_{1}(\mathbf{r})=\left(2 \pi \kappa^{2}\right)^{-1} \int d^{3} r^{\prime} \mathbf{j}\left(\mathbf{r}^{\prime}\right)\left|\mathbf{r}-\mathbf{r}^{\prime}\right|^{-1}$, which describes the vector potential induced by the currents $\mathbf{j}$ flowing in the superconductor (see Ref. 15 for more details). For thin superconducting cylindrical shells under consideration $(d<1$, $d \ll R, L)$, the vector potential $\mathbf{A}_{1}$ is proportional to the ratio $d / \kappa^{2}$, so that the results obtained for $\kappa=0.77$ and a given value $d$ can be applied also to cylinders with larger or smaller values of $\kappa$, provided that the quantity $d / \kappa^{2}$ remains the same.

\section{RESULTS AND DISCUSSION}

In the absence of an applied current, the vortices and antivortices ${ }^{17}$ induced in a cylindrical shell by a magnetic field 
(a)

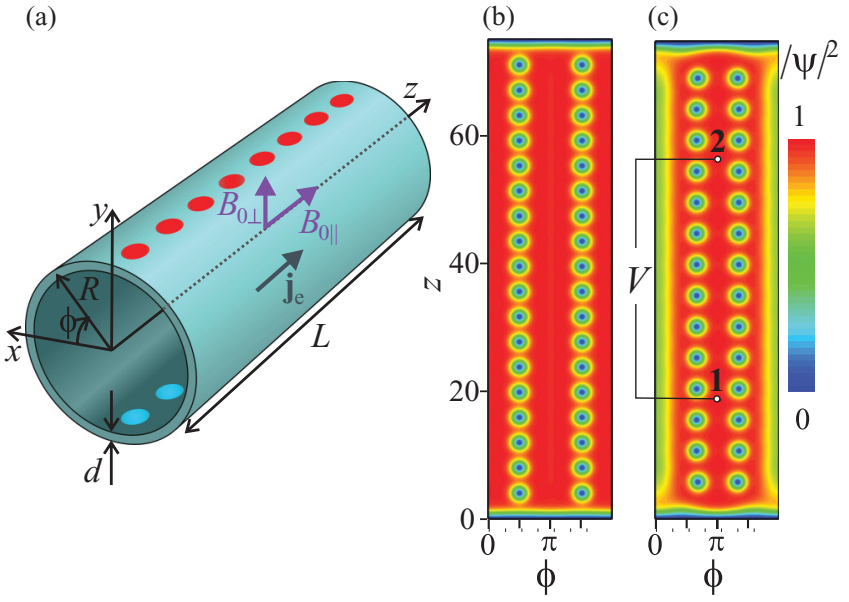

FIG. 1. (Color online) (a) Structure under consideration. The red spots at $\phi=\pi / 2$ (light blue spots at $\phi=3 \pi / 2$ ) schematically show equilibrium positions of vortices (antivortices), induced by the field $B_{0 \perp}$, in the case of $j_{e}=0$ and $B_{0 \|}=0$. (b) Equilibrium distribution of the square modulus of the order parameter in the hollow cylinder with $L=75, R=3$, and $d \rightarrow 0$ at $B_{0 \perp}=0.35, B_{0 \|}=0$, and $j_{e}=0$. (c) Same as in panel (b) but for $j_{e}=0.22$. The voltage drop $V$ is calculated between the points labeled as " 1 " and " 2 " and located at $z=L / 4$ and $z=3 L / 4$, respectively.

$B_{0 \perp}=0.35$, are "geometrically pinned" by a Magnus-Lorentz force $^{6}$ to $\phi=\pi / 2$ and $\phi=3 \pi / 2$, respectively [see Fig. 1(b)]. When applying a relatively low current density $j_{e}$ along the cylinder in the $z$ direction, vortices and antivortices experience a force towards $\phi=\pi$ where they can annihilate. Some of the vortices and antivortices do annihilate with each other, but most of them remain "pinned", although their equilibrium positions are somewhat shifted towards $\phi=\pi$ due to the Lorentz force proportional to the applied current [Fig. 1(c)]. At a certain current density, the system enters the dissipative regime: the vortex-antivortex pairs continuously recombine at $\phi=\pi$ while new pairs are nucleated at $\phi=0$, where the applied current, summed up with the Meisser current, significantly suppresses the order parameter [see Fig. 1(c)].

In Fig. 2(a) the critical current density $j_{1}$, corresponding to the onset of the resistive state in the middle part of the cylinder (between $z=L / 3$ and $z=3 L / 4$ ), is plotted as a function of the radius $R$ of the cylindrical shell at a fixed applied magnetic field, $B_{0 \perp}=0.9$ and $B_{0 \|}=0$. As seen from Fig. 2(a), the critical current density $j_{1}(R)$ for cylindrical shells is appreciably higher than that for the corresponding flat stripes with the same cross section and manifests a nonmonotonous behavior. As the radius is decreased (down to $R \approx 1.5$ ), the critical current density increases. This is due to an enhancement of the geometric pinning of vortices and antivortices as $R$ gets smaller. However, for $1.1<R<1.5$ the behavior of $j_{1}(R)$ becomes qualitatively different: it decreases with reducing $R$. This decrease is related to the fact that at those radii the distance between vortices, located at $\phi \approx \pi / 2$, and antivortices, located at $\phi \approx 3 \pi / 2$, becomes comparable to the vortex size, so that their annihilation is facilitated by reducing $R$. At $R<1.1$ even rather weak external current densities $j_{e}$ are sufficient for complete annihilation of vortex-antivortex pairs. In this case, the onset of a resistive state is determined
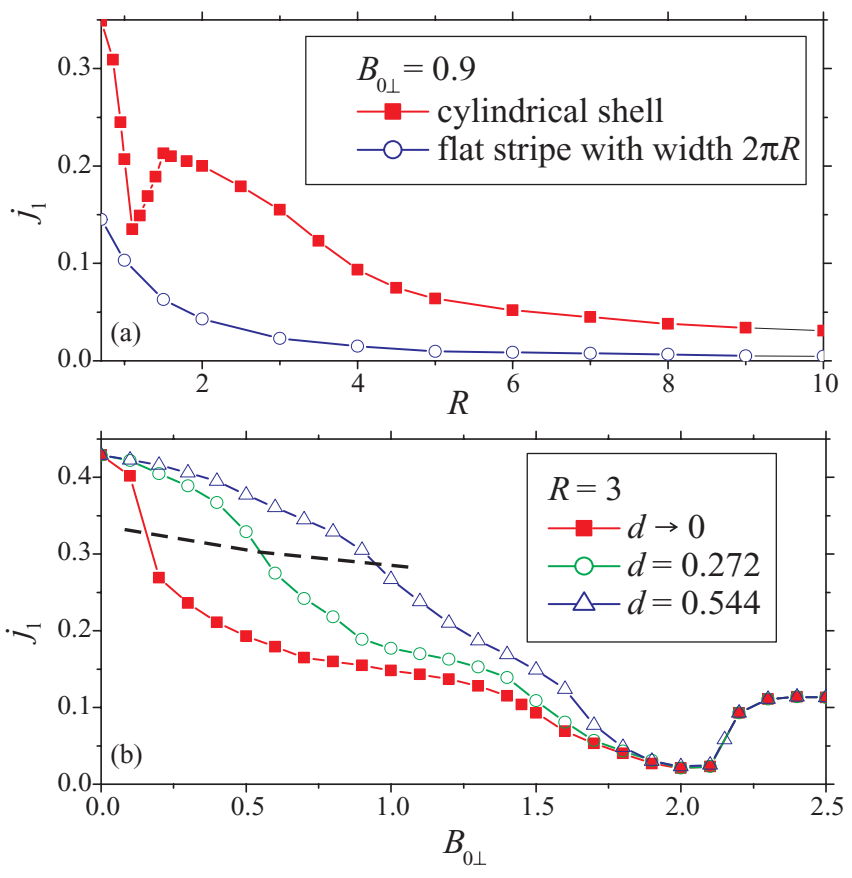

FIG. 2. (Color online) (a) Critical current density $j_{1}$ as a function of the cylinder radius $R$ at $L=50, d \rightarrow 0, B_{0 \perp}=0.9$, and $B_{0 \|}=0$. For comparison, the critical current density $j_{1}$ is shown also for a flat stripe with width $2 \pi R$, length $L=50$, and thickness $d \rightarrow 0$ in a perpendicular magnetic field $B_{0 \perp}=0.9$. (b) Critical current density $j_{1}$ as a function of $B_{0 \perp}$ at $R=3, L=50, B_{0 \|}=0$, and different $d$. The dashed line roughly shows the boundary between the ranges of parameters where the critical current density $j_{1}$ corresponds to the onset of vortex-antivortex nucleation and recombination (below the line) or to the transition from the Meissner state to the normal state (above the line).

by the nucleation of new pairs, which requires larger applied current densities $j_{e}$ at smaller $R$. As a result, in this range of $R$ the critical current density rapidly increases with decreasing $R$.

The calculated dependence of $j_{1}$ on the applied field $B_{0 \perp}$ [Fig. 2(b)] is qualitatively similar to that found in Ref. 8 in the limit $d \rightarrow 0$ and for applied fields below $H_{c 2}\left(B_{0 \perp}<2\right)$. Increasing the shell thickness $d$ leads to an increase in the critical current density $j_{1}$ due to partial screening of the applied field $B_{0 \perp}$ by supercurrents, so that a higher current must be applied in order to produce a Lorentz force sufficient for vortex/antivortex depinning. The shape of the curves $j_{1}\left(B_{0 \perp}\right)$ reveals several regimes.

(i) At relatively low magnetic fields, no vortices appear in the cylinder, so that the values of $j_{1}$ actually correspond to the transition from the Meissner state to the normal state.

(ii) With increasing applied magnetic field, the critical current density for nucleation of vortex-antivortex pairs becomes smaller than the depairing current density so that $j_{1}$ corresponds to the onset of vortex-antivortex nucleation, propagation, and annihilation.

(iii) At even higher fields, vortices and antivortices are present in the cylinder already at $j_{e}<j_{1}$. The critical current density decreases monotonously with increasing $B_{0 \perp}$ [both due to an increase of the depinning Lorentz force, proportional 
to $B_{0 \perp}$, and an enhanced mutual repulsion of the increasing number of vortices (antivortices)].

(iv) When approaching the second critical field $\left(B_{0 \perp}=2\right)$, the number and density of vortices (antivortices) become so large that the distances between the vortex cores are smaller than the vortex size. The resulting enhancement of mutual repulsion between vortices (antivortices) leads to a rather fast decrease of $j_{1}$ with $B_{0 \perp}$ in this field range. At $B_{0 \perp}=2$ the curves $j_{1}\left(B_{0 \perp}\right)$ exhibit a pronounced minimum.

(v) At $B_{0 \perp}>2$, the critical current density first sharply increases and then slowly decreases. In this field range, normal regions are formed in the cylinder around $\phi=\pi / 2$ and $\phi=3 \pi / 2$, and the vortex dynamics involves the entrance of vortices and antivortices from the normal regions to the superconducting regions, which requires relatively high applied current densities.

Obviously, only the range of parameters corresponding to regimes (ii) to (iv) can be relevant for revealing the effect of persistent currents, induced by the field $B_{0 \|}$, on vortex dynamics. As illustrated by Fig. 2, this range is sufficiently wide.

In Fig. 3 we plot the calculated time-averaged voltage drop $V$ between points 1 and 2 [see Fig. 1(a)] as a function of an increasing parallel magnetic field $B_{0 \|}$ for different values of the externally applied current density $j_{e}$. The accuracy of

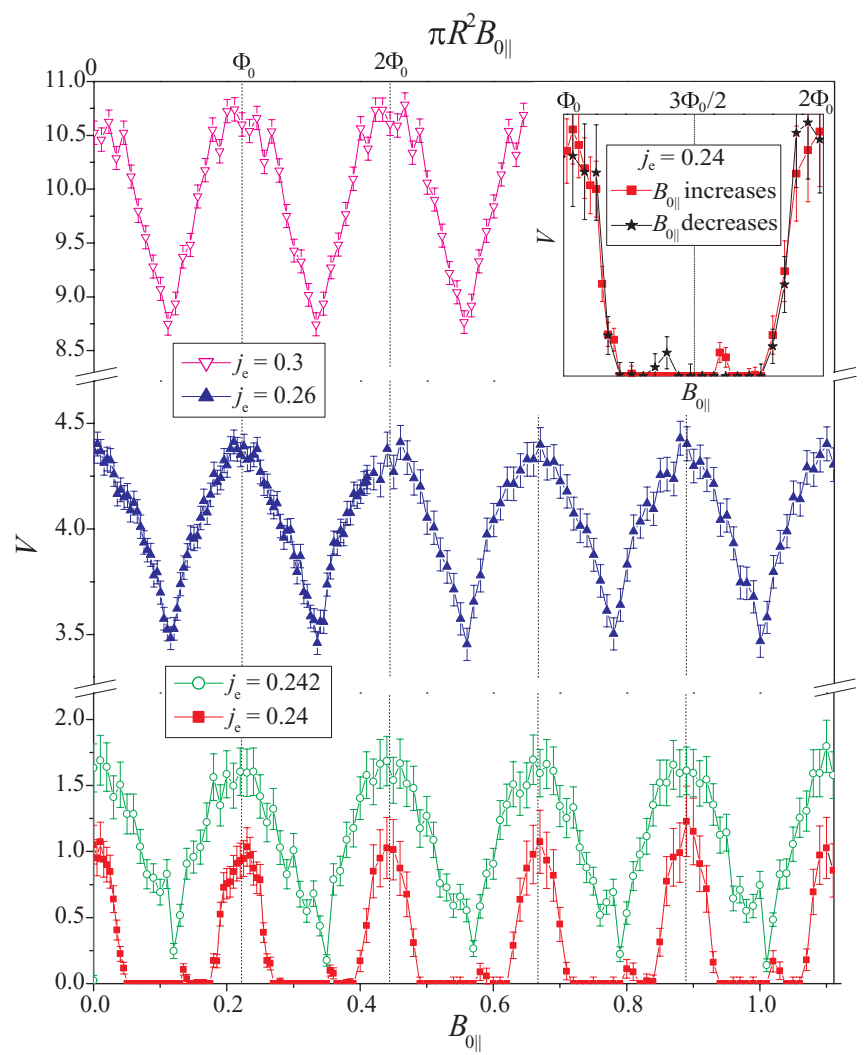

FIG. 3. (Color online) Time-averaged voltage drop $V$ between points 1 and 2 [see Fig. 1(c)] in a hollow cylinder with $L=75$, $R=3$, and $d \rightarrow 0$ as a function of an increasing magnetic field $B_{0 \|}$ for $B_{0 \perp}=0.35$ and different applied current densities $j_{e}$. Inset: Voltage drop $V$ as a function of the applied parallel magnetic field in the cases of increasing (squares) and decreasing (stars) field $B_{0 \|}$ for $L=75, R=3, d \rightarrow 0$, and $B_{0 \perp}=0.35$. the shown $V$ values is determined by a limited averaging time in the performed calculations. When switching $B_{0 \|}$ to a new value, a time interval $t_{\mathbf{r}}$, ranging from 100 to 500 , is reserved in the computational program for transient processes. Within this time interval, no calculation of $V$ is performed. Then the voltage drop $V$ is averaged over a time interval $t_{\text {aver }}$, ranging from 500 to 5000 in the present calculations. However, our analysis shows that in the multivortex system under consideration the full period of vortex-antivortex generation/recombination processes can be rather long, so that in general this period is not much smaller than the used time intervals $t_{\text {aver }}$. The error bars, shown in Fig. 3, correspond to the estimates, obtained using different values of $t_{\mathbf{r}}$ and $t_{\text {aver }}$.

As seen from Fig. 3, there are well-pronounced oscillations of the calculated voltage $V$ versus $B_{0 \|}$. The oscillation period is typical for the $\mathrm{AB}$ oscillations and equals $\Phi_{0} /\left(\pi R^{2}\right)$. The voltage $V$ takes maximum values for vanishing persistent currents induced by the field $B_{0 \|}$, i.e., for $\pi R^{2} B_{0 \|} \approx(2 n+$ 1) $\Phi_{0} / 2(n \in \mathbb{Z})$, while the sharp minima of $V$ approximately correspond to the maximum magnitude of the persistent currents, i.e., to $\pi R^{2} B_{0 \|}=(n+1 / 2) \Phi_{0}(n \in \mathbb{Z})$. In other words, the shape of these oscillations is "inverted" as compared to the Little-Parks oscillations. ${ }^{13}$ The oscillating behavior of $V\left(B_{0 \|}\right)$, shown in Fig. 3, can be explained in terms of the distortion of vortex/antivortex trajectories due to the Lorentz forces caused by the persistent currents and the magnetic field $B_{0 \perp}$. In Figs. 4(a) to 4(c), we plot the distributions of the streaming parameter $S=\left[\left(t_{2}-t_{1}\right)^{-1} \int_{t_{1}}^{t_{2}}\left(\partial|\psi|^{2} / \partial t\right)^{2} d t\right]^{1 / 2}$, introduced in $^{2}$ to visualize the vortex/antivortex motion. As implied by Fig. 4(a), in the case of $B_{0 \|}=0$, and hence in the absence of the corresponding persistent currents, the vortex/antivortex trajectories in the middle part of the cylinder follow a circular cross section of the cylinder, with vortices moving from $\phi=0$ to $\pi$ and antivortices moving from $\phi=2 \pi$ to $\pi$ all at the (a)

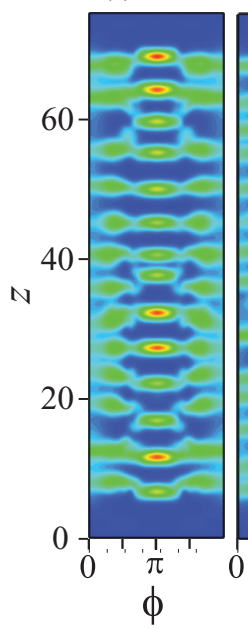

(b)

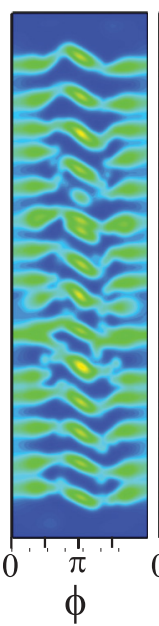

(c)

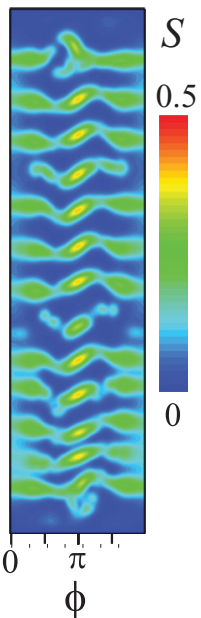

(d)

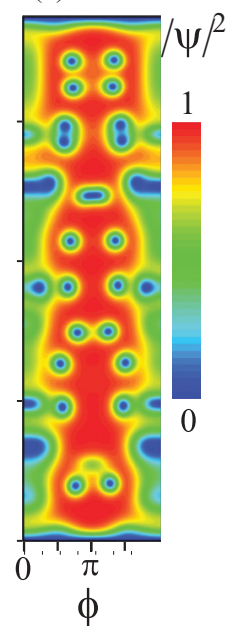

FIG. 4. (Color online) Panels (a) to (c): Distributions of the streaming parameter $S$ for $L=75, R=3, d \rightarrow 0, B_{0 \perp}=0.35$, $j_{e}=0.26, t_{2}-t_{1}=15$, and different values of the applied parallel magnetic field: $B_{0 \|}=0$ (a), $B_{0 \|}=0.1$ (b), and $B_{0 \|}=0.125$ (c). Panel (d): Snapshot of the square modulus of the order parameter in the cylindrical shell with $L=75, R=3$, and $d \rightarrow 0$ at $B_{0 \perp}=0.35$, $j_{e}=0.3$, and $B_{0 \|}=0.2$. 
same value of $z$. Vortex-vortex interactions cause only slight deviations from ideal circles. However, in the cases when the field $B_{0 \|}$ induces strong diamagnetic [ $B_{0 \|}=0.1$; see Fig. 4(b)] or paramagnetic $\left[B_{0 \|}=0.125\right.$; see Fig. $\left.4(\mathrm{c})\right]$ currents in the cylindrical shell, these currents cause a rather pronounced deformation of vortex/antivortex trajectories, so that the path of a vortex (antivortex) from $\phi=0(2 \pi)$ to $\phi=\pi$ partly follows the ellipse traced out by a slanted cross section of the cylinder. This represents an appreciably longer path than for $B_{0 \|}=0$.

The behavior of $V\left(B_{0 \|}\right)$ at the applied current density $j_{e}=0.24$, which only slightly exceeds the critical current density $j_{1}=0.2392$ at $B_{0 \|}=0$, implies that-in addition to oscillations of $V\left(B_{0 \|}\right)$ at a fixed $j_{e}$ value-also the critical current density $j_{1}$ is an oscillating function of the applied parallel magnetic field $B_{0 \|}=0$. However, our calculations show that the corresponding oscillation amplitudes are very small. For the parameters under consideration the critical current at $\pi R^{2} B_{0 \|}=(n+1 / 2) \Phi_{0}$ is $j_{1}=0.2416$, i.e., the oscillation amplitude for $j_{1}$ does not exceed $0.6 \%$. Thus we conclude that the voltage $V\left(B_{0 \|}\right)$ is a better indicator than the critical current to reveal the $\mathrm{AB}$ oscillations in vortex trajectories.

Besides pronounced oscillations of the voltage $V\left(B_{0 \|}\right)$ with period $\Phi_{0} /\left(\pi R^{2}\right)$, one can see in Fig. 3 several smaller features in the behavior of $V\left(B_{0 \|}\right)$. Many of those features are irregular and reflect the limited computation accuracy. However, some others are rather regular and periodic in $B_{0 \|}$. In particular, for $j_{1}=0.24$, small peaks of $V\left(B_{0 \|}\right)$ emerge at magnetic fields slightly above $B_{0 \|}=(n+1 / 2) \Phi_{0} /\left(\pi R^{2}\right)$. Those features can be attributed to the fact that the (time-averaged) number of vortex-antivortex pairs in the cylinder depends not only on the applied current density [see Figs. 1(b) and 1(c)] but also on the parallel field $B_{0 \|}$. At the same time, the time-averaged voltage drop $V$ as well as the critical current $j_{1}$ are, of course, sensitive to the number of vortices in the cylinder. This sensitivity leads, in particular, to the appearance of the peaks of $V\left(B_{0 \|}\right)$ for $j_{e}=0.24$ and $B_{0 \|}$ slightly above $(n+1 / 2) \Phi_{0} /\left(\pi R^{2}\right)$.

From Fig. 3, one can also observe that the curves $V\left(B_{0 \|}\right)$ are not fully symmetric with respect to the points $B_{0 \|}=$ $n \Phi_{0} /\left(\pi R^{2}\right)$ or $B_{0 \|}=(n+1 / 2) \Phi_{0} /\left(\pi R^{2}\right)$. Thus, in Fig. 3 , the aforementioned weak peaks of $V\left(B_{0 \|}\right)$ at $j_{1}=0.24$ are present at magnetic fields just above $B_{0 \|}=(n+1 / 2) \Phi_{0} /\left(\pi R^{2}\right)$, but no similar peaks appear below $B_{0 \|}=(n+1 / 2) \Phi_{0} /\left(\pi R^{2}\right)$. In our numerical simulations, we ramp up the magnetic field $B_{0 \|}$ and calculate how the order parameter adapts to the increased field. Geometric pinning of vortices in the cylinder leads to hysteresis effects and results in asymmetries in the curves. A similar pinning in superconducting spherical nanoshells has been shown to cause a pronounced hysteresis in the dependence of the number of vortex-antivortex pairs on the applied magnetic field. ${ }^{6}$ Also in the cylindrical shells under consideration the number of vortex-antivortex pairs and hence the curves $V\left(B_{0 \|}\right)$ demonstrate hysteretic behavior. The presence of such hysteretic behavior is illustrated in the inset of Fig. 3, where we compare the dependencies $V\left(B_{0 \|}\right)$, calculated for increasing and decreasing fields $B_{0 \|}$. As seen from this inset, in the case of a decreasing field $B_{0 \|}$, small peaks of $V\left(B_{0 \|}\right)$ appear to the left from $B_{0 \|}=(3 / 2) \Phi_{0} /\left(\pi R^{2}\right)$. Within the error bars, the whole pattern of $V\left(B_{0 \|}\right)$, where the field
$B_{0 \|}$ goes either up or down, looks symmetric with respect to $B_{0 \|}=(3 / 2) \Phi_{0} /\left(\pi R^{2}\right)$.

As further seen from Fig. 3, the shape of the curve $V\left(B_{0 \|}\right)$ becomes more regular and symmetric when increasing the applied current density to the value $j_{e}=0.26$, which is considerably higher than the critical current density $j_{1}$. However, relatively pronounced additional features, caused by variations of the (time-averaged) number of vortex-antivortex pairs in the cylinder, reappear in the curve $V\left(B_{0 \|}\right)$ at $j_{e}=0.3$ (Fig. 3), when—as illustrated by Fig. 4(d)—-some precursors of phase-slip-line formation can be already seen in the order parameter pattern. Remarkably, within the whole range of current densities considered here, the oscillation amplitude of $V\left(B_{0 \|}\right)$ remains appreciably large.

From Fig. 3, the magnitude of the resistivity oscillations is $\geqslant 0.05$ in the used units. This is about $0.5 \%$ of the normal-state resistivity, which equals 12 in our units, ${ }^{15}$ so that the predicted oscillations should be observable through four-probe measurements similar to those reported in Ref. 2. Our calculations show that the self-inductance of the cylinders under consideration has a relatively weak effect on the resistivity oscillations; as follows from Fig. 5(a), for $L=30, R=3, d=0.272, \kappa=0.77, B_{0 \perp}=0.6$, and
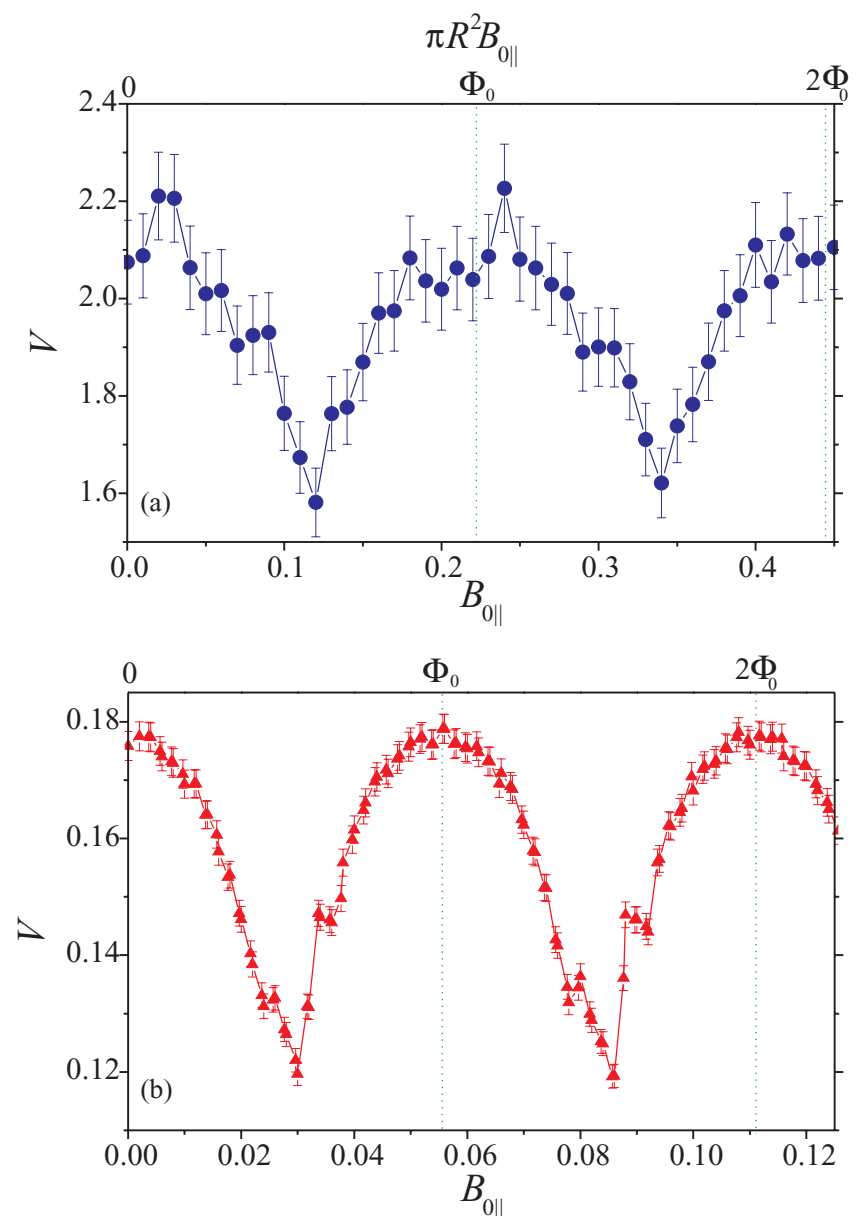

FIG. 5. (Color online) Time-averaged voltage drop $V$ as a function of an increasing magnetic field $B_{0 \|}$ in the cases of (a) $L=30$, $R=3, d=0.272, B_{0 \perp}=0.6$, and $j_{e}=0.28$ and (b) $L=30, R=6$, $d \rightarrow 0, B_{0 \perp}=0.2$, and $j_{e}=0.2$. 
$j_{e}=0.28$ the oscillation magnitude is about 0.07 . This magnitude tends to decrease when increasing the radius of the cylinder and/or when decreasing its length down to values $L<2 \pi R$ [cp. Fig. 5(b) to Fig. 3]. Nevertheless, for $L=30$, $R=6, d \rightarrow 0, B_{0 \perp}=0.2$, and $j_{e}=0.2$ our calculations predict an oscillation magnitude as large as 0.01 [see Fig. 5(b)]. Of course, in very short cylinders with $R \sim 1$, which cannot accommodate vortices, the predicted voltage oscillations become impossible.

It seems worth emphasizing that the material and geometric parameters, required to observe the predicted resistivity oscillations, are achievable experimentally. For example, with $\xi \approx 150 \mathrm{~nm}$ (close to the values of $\xi(0)$ for Al hollow cylinders in Ref. 2), the dimensionless parameters $R=3$ and $d=0.272$ would correspond to a hollow cylinder with a radius of about $640 \mathrm{~nm}$ (a few times larger than the cylinder radii in Ref. 2) and a wall thickness of about $60 \mathrm{~nm}$ (twice that in Ref. 2).

\section{CONCLUSIONS}

To conclude, we have shown that in hollow superconductor cylinders, subjected to a tilted magnetic field, the resistance, caused by vortex motion, should manifest measurable oscillations as a function of the magnetic field component parallel to the cylinder axis. This effect can provide a robust tool to probe experimentally the oscillating persistent currents, related to the $\mathrm{AB}$ effect, in a wide range of parameters, in particular, much below the superconducting critical temperature.

\section{ACKNOWLEDGMENTS}

This work was supported by Methusalem funding by the Flemish government; the Flemish Science Foundation (FWO-Vl), in particular FWO Projects No. G.0356.05, No. G.0115.06, No. G.0370.09N, and No. G.0115.12N; the Scientific Research Community, Project No. WO.033.09N; the Belgian Science Policy; and the ESF NES Network.
${ }^{1}$ Y. Liu, Yu. Zadorozhny, M. M. Rosario, B. Y. Rock, P. T. Carrigan, and H. Wang, Science 294, 2332 (2001).

${ }^{2}$ H. Wang, M. M. Rosario, N. A. Kurz, B. Y. Rock, M. Tian, P. T. Carrigan, and Y. Liu, Phys. Rev. Lett. 95, 197003 (2005).

${ }^{3}$ Q. Du and L. Ju, J. Comput. Phys. 201, 511 (2004).

${ }^{4}$ Q. Du and L. Ju, Math. Comput. 74, 1257 (2004).

${ }^{5}$ V. N. Gladilin, J. Tempere, I. F. Silvera, J. T. Devreese, and V. V. Moshchalkov, Phys. Rev. B 77, 024512 (2008).

${ }^{6}$ J. Tempere, V. N. Gladilin, I. F. Silvera, J. T. Devreese, and V. V. Moshchalkov, Phys. Rev. B 79, 134516 (2009).

${ }^{7}$ M. Lu-Dac and V. V. Kabanov, Phys. Rev. Lett. 105, 157005 (2010).

${ }^{8}$ P. Sabatino, G. Carapella, and G. Costabile, Supercond. Sci. Technol. 24, 125007 (2011).

${ }^{9}$ V. M. Fomin, R. O. Rezaev, and O. G. Schmidt, Nano Lett. 12, 1282 (2012).
${ }^{10}$ Y. Aharonov and D. Bohm, Phys. Rev. 115, 485 (1959).

${ }^{11}$ M. V. Berry, Proc. R. Soc. London A 392, 45 (1984).

${ }^{12}$ B. S. Deaver and W. M. Fairbank, Phys. Rev. Lett. 7, 43 (1961).

${ }^{13}$ W. A. Little and R. D. Parks, Phys. Rev. Lett. 9, 9 (1962).

${ }^{14}$ T.-C. Wei and P. M. Goldbart, Phys. Rev. B 77, 224512 (2008).

${ }^{15}$ A. V. Silhanek, V. N. Gladilin, J. Van de Vondel, B. Raes, G. W. Ataklti, W. Gillijns, J. Tempere, J. T. Devreese, and V. V. Moshchalkov, Supercond. Sci. Technol. 24, 024007 (2011).

${ }^{16} \mathrm{M}$. Tinkham, Introduction to Superconductivity, 2nd ed. (McGrawHill, New York, 1996).

${ }^{17}$ Like in Ref. 6, we use the term "vortex" ("antivortex") if the magnetic moment, induced by the corresponding circulating quasi2D supercurrents, is parallel (antiparallel) to the outer normal to the superconductor shell. 